\title{
Rapid diagnostic tests for malaria: past, present and future
}

\section{Juan José Valle-Delgado ${ }^{1}$ \& Xavier Fernàndez-Busquets ${ }^{\dagger 2-4}$}

${ }^{1}$ Department of Forest Products Technology, School of Chemical Technology, Aalto University, P.O. Box 16300, FI-00076 Aalto, Finland.

${ }^{2}$ Nanomalaria Group, Institute for Bioengineering of Catalonia (IBEC), Baldiri Reixac 10-12, ES-08028 Barcelona, Spain

${ }^{3}$ Barcelona Institute for Global Health (ISGlobal), Barcelona Center for International Health Research (CRESIB, Hospital Clínic-Universitat de Barcelona), Rosselló 149153, ES-08036 Barcelona, Spain

${ }^{4}$ Nanoscience and Nanotechnology Institute (IN2UB), University of Barcelona, Martí i Franquès 1, ES-08028 Barcelona, Spain

${ }^{\dagger}$ Author for correspondence: xfernandez_busquets@ ub.edu 
Prompt and accurate diagnosis of malaria is part of an effective disease management ${ }^{(1)}$, because if not treated malaria can quickly become life-threatening, whereas false positives increase treatment costs and drug-induced resistance, giving a wrong idea of therapeutic efficacy. Since the symptoms of malaria are nonspecific, the observation of clinical features alone might not be enough and should be confirmed with a parasitological analysis. Microscopic examination of Giemsa-stained thin and/or thick blood smears remains the conventional approach for diagnosis ${ }^{(2)}$. The sensitivity of this relatively inexpensive method is excellent, allowing the detection of as few as 5 parasites per $\mu \mathrm{L}$ of blood, and permitting also the determination of the infecting species and of the developmental stage of circulating parasites. In addition, smears provide a permanent record for quality assessment of the diagnosis. However, microscopy requires considerable expertise learned through extended training, the procedure is labor-intensive and time-consuming, and the variability in stains and in techniques used to collect and process blood affects slide interpretation ${ }^{(3)}$. Finally, routine clinical microscopy cannot reliably detect very low parasitemias $(<5$ parasites $/ \mu \mathrm{L})$ or sequestered parasites, and mixed infections are often missed, especially when Plasmodium malariae and Plasmodium ovale are present, as their densities are often low relative to Plasmodium falciparum ${ }^{(4)}$.

Alternative methods to microscopy include immunochromatographic analyses based on the detection of some antigens either specific to a Plasmodium species like $P$. falciparum histidine-rich protein 2 (HRP2), or conserved across all human malarias such as Plasmodium lactate dehydrogenase and aldolase. Most of these approaches have led to commercially available rapid diagnostic tests (RDTs) ${ }^{(5)}$, which have the advantages of being fast and simple without requiring extensive training or equipment. The results of the test should be rapidly available while the physician is actively 
managing patients, typically in less than one hour ${ }^{(6)}$, and the stability of RDT components should be such that refrigeration and a cold chain are not needed, whereas storage shelf life should be of sufficient long duration to minimize the logistical burden of resupply. A RDT strip chip has been designed for diagnosis of malaria infection ${ }^{(7)}$, where different lines clearly visible to the naked eye indicate the presence of specific antigens in blood. Developments in nanoparticle-based immunochromatographic RDTs reached modest sensitivities of $\leq 500 P$. falciparum $/ \mu \mathrm{L}^{(8)}$, although a test has been successfully evaluated for field use ${ }^{(9)}$. Most current RDTs, though, involve some important limitations ${ }^{(10)}$, such as the inability to simultaneously detect multiple strains and a too high detection threshold when considering the low level of parasitemia for immune populations, as well as for patients treated with antimalarial chemoprophylaxis. These gaps point out the need to develop new simple and rapid diagnostic tools showing higher sensitivity, selectivity and accuracy. Such a new generation of diagnostic devices should enable prediction of the therapeutic response at the beginning of a treatment, avoid the problems related to false positives and negatives, and be also capable of characterizing the precise infective Plasmodium species and strain through multiplexed measurements for the identification of parasite-specific antigens or enzymes.

Malaria parasites inside Plasmodium-infected red blood cells (pRBCs) grow hemozoin crystals which under an external magnetic field induce concentrationdependent optical dichroism. These magneto-optic properties have been harnessed for parasite detection in blood and tissues with low interference of other biological compounds ${ }^{(11)}$. More recently, PCR-based point-of-care tests have been adapted for the detection of low levels of strain-specific parasites ${ }^{(12)}$, even with saliva and urine as alternative DNA sources ${ }^{(13)}$, although their use is very limited in resource-poor settings. The application of lab-on-a-chip (LOC) technology ${ }^{(14)}$ could introduce a significant 
improvement in rapid malaria diagnosis, parasitemia quantification, and monitoring of the evolution of the disease and of the effect of drugs. This technology is based on devices of small dimensions incorporating micro- and nanotechnologies able to perform several analytical laboratory operations. LOCs exploit microfluidic effects to allow an expansion of the analyte detection range, increased sensitivity, and reduction of the required sample and reagent amounts. In addition to performing most of the analytical operations in a safe and contamination-free environment, LOC devices can be made with cheap polymeric materials adequate for low cost and disposable tests. Efficacious portable on-the-field diagnostic tools can be assembled combining LOC cartridges, where the blood sample would be introduced, with simple electronics to actuate them and read out the test results.

Microfluidics-based technology has potential for the design of point-of-care malaria diagnosis ${ }^{(15)}$. It has been suggested that in some cases the capillary blockage induced by $\mathrm{pRBCs}$ in severe malaria is due to loss of cell deformability ${ }^{(16)}$, likely triggered by an alteration in surface charge and plasticity of infected cells, a phenomenon which allows separating non-parasitized RBCs from pRBCs using dielectrophoretic forces ${ }^{(17)}$. Free flow electrophoresis is a continuous separation and preparative technique where an electric field is applied perpendicular to a sample stream that is continuously pumped into a separation cavity, which has been shown to be useful in a number of applications including cell sorting. The separation of $\mathrm{pRBCs}$ from RBCs is possible due to the alteration of the electrical conductivity of erythrocyte membranes, which significantly increases when RBCs become host to malaria parasites ${ }^{(18)}$. Free flow electrophoresis using a microfluidic system could provide an ideal platform for the development of a pRBC sorter by exploiting the advantages of low sample amounts and fast analysis times. Many groups are focusing their research on this type of devices, but 
progress is still needed to reduce costs; one example is the European Commission (EC)funded NanoMal project ${ }^{(19)}$, which is developing a simple, rapid and affordable pointof-care handheld system for malaria diagnosis and detection of drug resistant parasites within minutes. Another EC-funded project is DiscoGnosis ${ }^{(20)}$, where a disc-shaped plastic disposable chip handles a blood sample which is distributed among integrated chambers using centrifugal forces and microfluidic unit operations; all necessary (bio)chemicals are pre-stored in the disc, together with micro/nanocomponents, for fully automated analysis.

An interesting alternative approach is based on the isothermal conversion of single DNA cleavage-ligation events, catalyzed specifically by the Plasmodium enzyme topoisomerase I, to products detected at the individual molecule level ${ }^{(21)}$. This strategy allowed, combined with a droplet microfluidics LOC platform, for sensitive, specific, and quantitative detection of less than one parasite $/ \mu \mathrm{L}$ of all human malaria-causing Plasmodium species in single drops of unprocessed blood. The setup also allowed for detection of parasites in noninvasive saliva samples from infected patients. In terms of detection limit this assay outcompetes state-of-the-art light microscopy ( 5 parasites $/ \mu \mathrm{L}$ ) and RDTs ( $\geq 200$ parasites/ $\mu \mathrm{L})$, whereas in comparison to PCR, which is the only method with comparable sensitivity, the LOC system described is directly quantitative and requires no sample preparation. However, LOC-based devices require unambiguous and automated read-out formats and a simplified, pumpless syringe-based inlet system not available yet. Nevertheless, even in the current proof-of-concept setup, time-toreadout (ca. $2.5 \mathrm{~h})$ and price ( $c a .2 \mathrm{USD} / \mathrm{sample})$ suggest that such prototypes might be acceptable for routine use ${ }^{(21)}$. Last but not least, since topoisomerase I is an essential enzyme conserved across all Plasmodium species, the above described LOC platform might be developed into a highly sensitive and specific pan-malaria test, in contrast to 
many of the common commercially available RDTs specific for biomarkers such as HRP2, which detect P. falciparum only and are further hampered in regions where hrp2 genes are not expressed in a significant proportion of parasites ${ }^{(22)}$. On the other hand, such universal detection of Plasmodium goes against the need of diagnosis systems capable of rapidly discriminating different species and strains.

A low-cost microfluidic LOC for rapid genotyping of malaria-transmitting mosquitoes has also been recently described ${ }^{(23)}$. Several dozen Anopheles species are vectors of Plasmodium, some of which are difficult to distinguish morphologically, although this is fundamental to the development of control strategies and epidemiological studies of disease transmission. The inexpensive ( $<2 \mathrm{USD} /$ test $)$, disposable, field-deployable, and sample-to-answer microfluidic chip was developed for rapid molecular identification of Anopheles gambiae and Anopheles arabiensis using three isothermal DNA amplification reactors where crushed mosquito tissue was fed. The specific amplification products were labeled with an intercalating fluorescent dye whose emitted light was observed by eye and could be recorded with a cell-phone camera, which can be used for record keeping, transfer of data to a central processing facility, and for the provision of space and time stamps.

Because the sulfated polysaccharide heparin and other glycosaminoglycans (GAGs) have been demonstrated to bind pRBCs with high specificity and affinity in comparison to $\mathrm{RBCs}^{(24,25,26)}$, this property can potentially be harnessed for the design of novel RDTs based on GAG-pRBC recognition. In a preliminary assay, heparin was micropatterned onto perfluoropolyether-based polymers via photobiotin activation ${ }^{(27)}$, although these arrays did not provide sufficiently specific pRBC recognition. The main identified risks were: (i) developing an adequate immobilizing strategy of GAGs on solid substrates, which will likely involve the use of linkers and an important chemistry 
work; (ii) designing a method for sample treatment in order to expose to GAG-coated areas in the chip whole pRBCs or pRBC membranes, with the capability of washing away unbound components; and (iii) establishing a simple protocol for increasing sample signal with the objective of improving the sensitivity for the detection of low parasitemias.

If these pitfalls can be successfully dealt with, GAG-based RDTs would have several advantages relative to current antibody-based RDTs: (i) GAGs are more sturdy than proteins (antibodies) in front of the harsh environmental conditions found in malaria endemic regions; foreseeingly, a GAG-based RDT will not require cold storage and can last for months at temperatures close to $40{ }^{\circ} \mathrm{C}$ if adequately stored dry and in the dark. (ii) GAG production is more economically affordable than that of antibodies. (iii) Given the absence of known resistances of Plasmodium to heparin, it can be speculated that the antimalarial mechanism of sulfated polysaccharides has some component based on complex interactions with the pRBC membrane; if so, a GAGbased RDT might be used for pan-malaria diagnosis and would not be so susceptible to the elevated clonal variability of the parasite as antibody-based devices based on the recognition of small antigenic determinants.

\section{References}

1. World Health Organization: Guidelines for the treatment of malaria. 3rd Edition. http://apps.who.int/iris/bitstream/10665/162441/1/9789241549127_eng.pdf (2015).

2. Wongsrichanalai C, Barcus MJ, Muth S, Sutamihardja A, Wernsdorfer WH: A review of malaria diagnostic tools: microscopy and rapid diagnostic test (RDT). Am.J.Trop.Med.Hyg. 77(6 Suppl), 119-127 (2007). 
3. Warhurst DC, Williams JL: Laboratory diagnosis of malaria. J.Clin.Pathol. 49, 533-538 (1996).

4. Kilian AHD, Metzger WG, Mutschelknauss EJ et al: Reliability of malaria microscopy in epidemiological studies: results of quality control. Trop.Med.Int.Health 5(1), 3-8 (2000).

5. Moody A: Rapid diagnostic tests for malaria parasites. Clin.Microbiol.Rev. 15(1), 66-78 (2002).

6. Murray CK, Gasser RA, Magill AJ, Miller RS: Update on rapid diagnostic testing for malaria. Clin.Microbiol.Rev. 21(1), 97-110 (2008).

7. Bell D, Wongsrichanalai C, Barnwell JW: Ensuring quality and access for malaria diagnosis: how can it be achieved? Nat.Rev.Microbiol. 4(9 Suppl), S7-20 (2006).

8. Hirsch LR, Halas NJ, West JL: Whole-blood immunoassay facilitated by gold nanoshell-conjugate antibodies. Methods Mol.Biol. 303, 101-111 (2005).

9. Polpanich D, Tangboriboonrat P, Elaissari A, Udomsangpetch R: Detection of malaria infection via latex agglutination assay. Anal.Chem. 79(12), 4690-4695 (2007).

10. Hauck TS, Giri S, Gao Y, Chan WCW: Nanotechnology diagnostics for infectious diseases prevalent in developing countries. Adv.Drug Deliv.Rev. 62, 438-448 (2010).

11. Newman DM, Heptinstall J, Matelon RJ et al: A magneto-optic route toward the in vivo diagnosis of malaria: preliminary results and preclinical trial data. Biophys.J. 95(2), 994-1000 (2008).

12. Mahajan B, Zheng H, Pham PT et al: Polymerase chain reaction-based tests for pan-species and species-specific detection of human Plasmodium parasites. Transfusion 52(9), 1949-1956 (2012). 
13. Putaporntip C, Buppan P, Jongwutiwes S: Improved performance with saliva and urine as alternative DNA sources for malaria diagnosis by mitochondrial DNAbased PCR assays. Clin.Microbiol.Infect. 17(10), 1484-1491 (2011).

14. Huikko K, Kostiainen R, Kotiaho T: Introduction to micro-analytical systems: bioanalytical and pharmaceutical applications. Eur.J.Pharm.Sci. 20(2), 149-171 (2003).

15. Rodrigues Ribeiro Teles FS, Pires de Távora Tavira LA, Pina da Fonseca LJ: Biosensors as rapid diagnostic tests for tropical diseases. Crit.Rev.Clin.Lab.Sci. 47(3), 139-169 (2010).

16. Shelby JP, White J, Ganesan K, Rathod PK, Chiu DT: A microfluidic model for single-cell capillary obstruction by Plasmodium falciparum-infected erythrocytes. Proc.Natl.Acad.Sci.U.S.A. 100(25), 14618-14622 (2003).

17. Gascoyne P, Mahidol C, Ruchirawat M, Satayavivad J, Watcharasit P, Becker FF: Microsample preparation by dielectrophoresis: isolation of malaria. Lab Chip 2(2), 70-75 (2002).

18. Gascoyne P, Satayavivad J, Ruchirawat M: Microfluidic approaches to malaria detection. Acta Trop. 89(3), 357-369 (2004).

19. Nanomal Project. http://www.nanomal.org/ (2013).

20. DiscoGnosis Project. http://www.discognosis.eu (2013).

21. Juul S, Nielsen CJF, Labouriau R et al: Droplet microfluidics platform for highly sensitive and quantitative detection of malaria-causing Plasmodium parasites based on enzyme activity measurement. ACS Nano 6(12), 10676-10683 (2012).

22. Bronzan RN, McMorrow ML, Kachur SP: Diagnosis of malaria: challenges for clinicians in endemic and non-endemic regions. Mol.Diagn.Ther. 12, 299-306 (2008). 
23. Liu C, Mauk MG, Hart R, Bonizzoni M, Yan G, Bau HH: A low-cost microfluidic chip for rapid genotyping of malaria-transmitting mosquitoes. PLOS ONE 7(8), e42222- (2012).

24. Marques J, Vilanova E, Mourão PAS, Fernàndez-Busquets X: Marine organism sulfated polysaccharides exhibiting significant antimalarial activity and inhibition of red blood cell invasion by Plasmodium. Sci.Rep. 6, 24368- (2016).

25. Agrawal MR, Ozarkar AD, Gupta S, Deobagkar DN, Deobagkar DD: Comparative study of Plasmodium falciparum erythrocyte membrane protein 1DBL $\alpha$ domain variants with respect to antigenic variations and docking interaction analysis with glycosaminoglycans. Mol.Biosyst. 10(9), 2466-2479 (2014).

26. Valle-Delgado JJ, Urbán P, Fernàndez-Busquets X: Demonstration of specific binding of heparin to Plasmodium falciparum-infected vs non-infected red blood cells by single-molecule force spectroscopy. Nanoscale 5(9), 3673-3680 (2013).

27. Credi C, De Marco C, Molena E et al: Heparin micropatterning onto foulingrelease perfluoropolyether-based polymers via photobiotin activation. Colloids Surf B Biointerfaces 146, 250-259 (2016). 